\title{
Infected Internal Pulse Generator of a Spinal Cord Stimulator Device Treated Successfully without Removal: A Case Report
}

Sir,

Implantation of a spinal cord stimulator (SCS) is an evidence-based treatment for severe and chronic neuropathic pain which has grown rapidly over the past four decades. One of the serious but devastating complications of this neuromodulatory technique is infection, and in the majority of cases, the removal of the device is required, despite appropriate antibiotic therapy. ${ }^{[1]}$ We describe our experience on a patient with SCS in whom wound infection and explantation of the SCS implantable pulse generator (IPG) was successfully disinfected in situ by the combined use of antibiotics and relocation of the device.

A 54-year-old male with a medical history of failed back surgery syndrome after previous laminectomies with posterior instrumentation underwent an epidural SCS implantation consisting of one 16 contact paddle lead (Specify $2 \times 8$ - 39286, Medtronic), two-lead extensions (Medtronic model 3708140), and a rechargeable IPG (IPG, Restore Ultra - 37712, Medtronic, Minneapolis, MN, USA), 7 years ago [Figure 1].

Eighteen months ago, he had a routine replacement of IPG due to battery depletion under local anesthesia and sedation. The new IPG (Restore Ultra - 37712, Medtronic) was inserted into the previous pocket placed in his right superior gluteal region, and the surgical wound was typically closed in two layers. He received two doses of teicoplanin $400 \mathrm{mg} \mathrm{IV}$, the postoperative course was uneventful, and he was discharged on the next day. On removing of skin stitches at a peripheral



Figure 1: Postoperative X-ray (anteroposterior view) of a $2 \times 8$ surgical paddle lead placed at T11-T12 connected to a rechargeable implantable pulse generator hospital 12 days later, a clean wound disruption of 2 -cm length was detected. There were no subcutaneous collections or signs of local infection. The patient was afebrile with no local tenderness or systemic symptoms, and the resident of emergencies restored the wound with nylon 3.0, after meticulous asepsis and debridement of skin edges.

He returned to our department 6 days later due to local tender and wetting of gauzes; the recently reapproximated skin edges showed signs of local inflammation with wound dehiscence and pus discharge. A $2 \mathrm{~cm} \times 2 \mathrm{~cm}$ portion of the generator hardware was extruding from his right superior buttock with sizeable erythema around the site [Figure 2]. However, the patient was in a good physical state and afebrile. Neurological examination was normal; however, initial laboratory tests revealed an increase in erythrocyte sedimentation rate and $\mathrm{C}$-reactive protein. Empirical treatment with intravenous vancomycin $(500 \mathrm{mg} / 6 \mathrm{~h})$ and cefepime $(2 \mathrm{gr} / 12 \mathrm{~h})$ was immediately started. Wound discharge was sent to pathology for cultures, which revealed Staphylococcus aureus with high sensitivity to the above antibiotics.

As the patient's clinical condition was stable and he was unwilling to undergo a late replacement of SCS device if the present one had to be removed, an attempt was made to try to preserve it. Therefore, after consent was obtained from the patient to maintain the implanted system and detailed discussion with infectologists, he underwent surgical exploration of the wound under general anesthesia. Upon entrance into IPG capsule, plenty

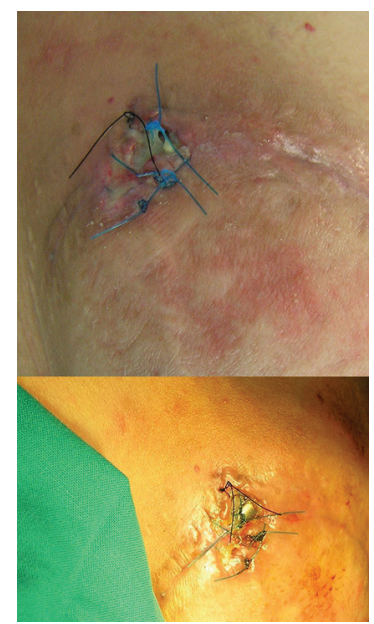

Figure 2: Local infection and extrusion of the implantable pulse generator 18 days' postimplantation 
of purulent material was encountered. The IPG was removed from the subcutaneous pocket and disconnected from the lead extensions. The lead extensions were also disconnected from the epidural electrode and removed. Impedances of the paddle lead were checked by the company representatives and were found adequate.

The IPG was meticulously cleaned and disinfected. First, it was several times rinsed in $0.9 \%$ of normal saline solution and later placed in a hydrogen peroxide solution for $15 \mathrm{~min}$. Afterward, the IPG scrubbed for $20 \mathrm{~min}$ using an aqueous $10 \%$ povidone-iodine solution, followed by soaking in a vancomycin solution ( $1 \mathrm{~g}$ of vancomycin powder dissolved in $20 \mathrm{ml}$ of normal saline) for another $20 \mathrm{~min}$. Finally, the IPG was taken off the vancomycin solution and vigorously rinsed again in normal saline. Gram staining of cultures taken from the IPG at this point was negative. The generator device was then repositioned in a new subcutaneous pocket, on the left superior gluteal region, opposite to the site of the original placement. Two new lead extenders were then subcutaneously tunneled on the left lumbar area and attached to the paddle lead proximally and to the IPG distally.

The pocket wound was also copiously irrigated with normal saline, then with hydrogen peroxide solution, followed by povidone-iodine and vancomycin powder solution, and finally was scrapped with a scalpel until new vital red tissue was observed. The skin surrounding the area of wound dehiscence and generator extrusion was resected. A flat drain was placed, and the wound was loosely approximated.

The intravenous administration of vancomycin and cefepime was continued for a 4-week period, and the patient was treated with oral rifampin for the next 2 months. There were no postoperative complications or implant system failure occurred. The cultures taken from the removed IPG after "disinfected toilette" were negative. At the time of writing this study, our patient is still well $1 \frac{1}{2}$ year after completion of therapy, free of local or systemic infection, showing a continuing therapeutic result with SCS which continues to work properly.

Although infection of a neuromodulatory system most often necessitates the removal of the device, SCS IPG may be salvaged in selected patients with mild clinical symptomatology. ${ }^{[2-4]}$ With this approach, some patients may be spared of SCS therapy interruptions and/ or additional procedures. Moreover, the replacement of such an expensive device increases medical costs in general and impacts negatively on the healthcare systems. To the best of our knowledge, this is the first reported case in the literature of a successfully SCS IPG infection treatment without removal of the system or discontinuation of therapy.

\section{Declaration of patient consent}

The authors certify that they have obtained all appropriate patient consent forms. In the form, the patient has given his consent for his images and other clinical information to be reported in the journal. The patient understand that name and initials will not be published and due efforts will be made to conceal identity, but anonymity cannot be guaranteed.

\section{Financial support and sponsorship}

Nil.

\section{Conflicts of interest}

There are no conflicts of interest.

\section{Aristedis Rovlias}

Department of Neurosurgery, Asclepeion General Hospital of Athens, Athens, Greece

Address for correspondence: Dr. Aristedis Rovlias, Department of Neurosurgery, Asclepeion General Hospital of Athens, 1 Vasileos Pavlou Street, 16 673, Voula, Athens, Greece. E-mail: arovlias@yahoo.com

\section{REFERENCES}

1. Yusuf E, Bamps S, Ursi JP, Del Biondo E, De Smedt K, Van Paesschen R, et al. Characteristics of infections associated with a spinal cord stimulator system. J Infect 2016;73:515-7.

2. Bendersky D, Yampolsky C. Is spinal cord stimulation safe? A review of its complications. World Neurosurg 2014;82:1359-68.

3. Eldabe S, Buchser E, Duarte RV. Complications of spinal cord stimulation and peripheral nerve stimulation techniques: A Review of the literature. Pain Med 2016;17:325-36.

4. Rabi J, Anitescu M. Late extrusion of an implantable pulse generator of a spinal cord stimulator. Pain Physician 2016;19:E671-4.

This is an open access journal, and articles are distributed under the terms of the Creative Commons Attribution-NonCommercial-ShareAlike 4.0 License, which allows others to remix, tweak, and build upon the work non-commercially, as long as appropriate credit is given and the new creations are licensed under the identical terms.

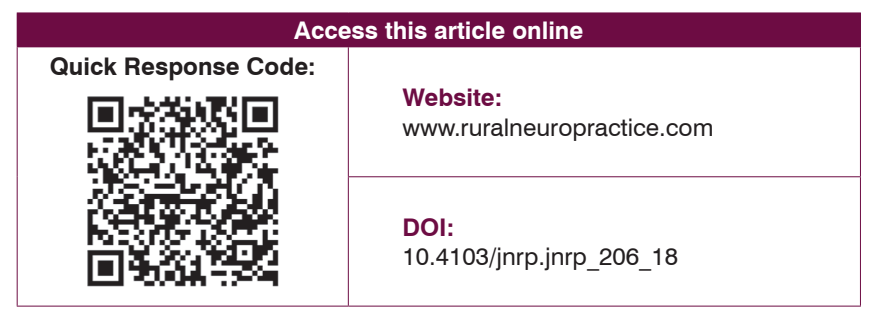

How to cite this article: Rovlias A. Infected internal pulse generator of a spinal cord stimulator device treated successfully without removal: A case report. J Neurosci Rural Pract 2019;10:168-9.

(c) 2018 Journal of Neurosciences in Rural Practice | Published by Wolters Kluwer - Medknow 